\title{
Significance of osteopontin in the sensitivity of malignant pleural mesothelioma to pemetrexed
}

\author{
SUSUMU TAKEUCHI ${ }^{1}$, MASAHIRO SEIKE ${ }^{1}$, RINTARO NORO ${ }^{1}$, CHIE SOENO $^{1}$, TEPPEI SUGANO ${ }^{1}$, \\ FENFEI ZOU $^{1}$, HARUKA UESAKA ${ }^{2}$, NOBUHIKO NISHIJIMA ${ }^{1}$, MASARU MATSUMOTO ${ }^{1}$, \\ YUJI MINEGISHI ${ }^{1}$, KAORU KUBOTA ${ }^{1}$ and AKIHIKO GEMMA ${ }^{1}$ \\ ${ }^{1}$ Department of Pulmonary Medicine and Oncology, Graduate School of Medicine, \\ Nippon Medical School, Tokyo 113-8603; ${ }^{2}$ MediBIC Group, Tokyo 102-8578, Japan
}

Received January 24, 2014; Accepted March 10, 2014

DOI: 10.3892/ijo.2014.2370

\begin{abstract}
Pemetrexed (PEM) is currently recommended as one of the standard anticancer drugs for malignant pleural mesothelioma (MPM). However, the mechanism of the sensitivity of MPM to PEM remains unclear. We analyzed the antitumor effects of PEM in six MPM cell lines by MTS assay. To identify genes associated with drug sensitivity, we conducted gene expression profiling on the same set of cell lines using GeneChips and pathway analysis. Three cell lines were sensitive to PEM. A total fo 18 transcripts and 14 genes identified by GeneChips were significantly correlated with sensitivity to PEM. Pathway analysis revealed that osteopontin (SPP1/OPN) was an important target in PEM sensitivity. Overexpression of SPP1/OPN was observed in the sensitive cells by quantitative PCR and western blot analysis. Introduction of SPP1/OPN by lentiviral vector significantly enhanced the invasion activities of MPM cells. PEM treatment with SPP1/OPN knockdown inhibited the PEM-induced cell growth-inhibitory effect in PEM-sensitive cells. Expression of SPP1/OPN and AKT phosphorylation significantly decreased after PEM treatment of the PEM-sensitive cells. High immunohistochemical expression of SPP1/OPN was observed in two of three MPM patients who had a partial response to PEM-based chemotherapy. PEM has antitumor effects in MPM cells dependent on SPP1/OPN overexpression resulting in AKT activation. Our results suggest that SPP1 may be used as a single predictive biomarker of the effectiveness of PEM treatment in MPM.
\end{abstract}

\section{Introduction}

Malignant pleural mesothelioma (MPM) is an aggressive tumor that arises from serosal cells with poor prognosis. The incidence of the disease is expected to steadily increase

Correspondence to: Dr Masahiro Seike, Department of Pulmonary Medicine and Oncology, Graduate School of Medicine, Nippon Medical School, 1-1-5, Sendagi, Bunkyo-ku, Tokyo 113-8603, Japan E-mail: mseike@nms.ac.jp

Key words: mesothelioma, pemetrexed, osteopontin, SPP, sensitivity because of occupational asbestos exposure and be recognized as a social problem in Japan (1). Over 100,000 MPM-related deaths are predicted to occur in the next 40 years in Japan (2).

Pemetrexed (PEM) is a multitarget antifolate agent and recently became a component of the standard therapy for MPM. In a phase III study, chemotherapy of PEM and cisplatin (CDDP) yielded the best effect for MPM in terms of response rate (41\%) and median survival time (12 months) (3). However, the major concern of this treatment is short duration of response. Development of resistance to drugs is a major impediment to the success of PEM-based chemotherapy. Elucidating the mechanism of the sensitivity of MPM to PEM would be crucial in improving the therapeutic strategies against MPM. Administration of PEM therapy to selected patients based on a specific biomarker is one strategy that could lead to improved MPM treatments.

In this study, we analyzed the antitumor effects of PEM in MPM cell lines. Gene expression analysis on the same set of cell lines was performed to identify the molecules associated with the sensitivity of MPM to PEM treatment. We ultimately identified osteopontin (SPP1/OPN) as a therapeutic biomarker for PEM treatment.

\section{Materials and methods}

Cell culture. We used the following 6 MPM cell lines in this study: NCI-H28, NCI-H2452 and ACC-MESO4 epithelial-type cell lines, NCI-H2052 sarcomatoid-type cell line, ACC-MESO1 fibrous cell line, and MSTO-211H biphasic-type cell line. NCI-H28, NCI-H2452, NCI-H2052 and MSTO-211H were purchased from American Type Culture Collection (Manassas, VA). ACC-MESO1 and ACC-MESO4 were obtained from the Riken Cell Bank (Tsukuba, Japan). MPM cells were maintained in RPMI-1640 (Gibco-BRL, Carlsbad, CA) with $10 \%$ fetal bovine serum.

Clinical samples. We evaluated tumor specimens from MPM patients who had been treated with combination therapy of PEM with platinum agents including CDDP and carboplatin (CBDCA) from 2007 to 2011 at Nippon Medical School Hospital. Tumor specimens were available from 6 patients. Three patients were judged as having partial response (PR) to the PEM-based 
Table I. $\mathrm{IC}_{50}$ values in 6 MPM cell lines responding to treatments with PEM, GEM and CDDP by MTS assay.

\begin{tabular}{lcccccc}
\hline $\begin{array}{l}\text { Cell line } \\
\text { Pathological type }\end{array}$ & $\begin{array}{c}\mathrm{H} 28 \\
\text { Epithelial type }\end{array}$ & $\begin{array}{c}211 \mathrm{H} \\
\text { Biphasic type }\end{array}$ & $\begin{array}{c}\mathrm{H} 2052 \\
\text { Sarcoma type }\end{array}$ & $\begin{array}{c}\text { H2452 } \\
\text { Epithelial type }\end{array}$ & $\begin{array}{c}\text { MESO1 } \\
\text { Fibrous type }\end{array}$ & $\begin{array}{c}\text { MESO4 } \\
\text { Epithelial type }\end{array}$ \\
\hline PEM IC $_{50}(\mu \mathrm{M})$ & $0.07 \pm 0.02$ & $0.07 \pm 0.01$ & $0.57 \pm 0.34$ & $>100$ & $>100$ & $>100$ \\
CDDP IC $_{50}(\mu \mathrm{M})$ & $89.4 \pm 12.4$ & $79.5 \pm 17.8$ & $57.9 \pm 6.8$ & $>100$ & $>100$ & $45.5 \pm 9.5$ \\
GEM IC $_{50}(\mu \mathrm{M})$ & $6.0 \pm 1.4$ & $53.9 \pm 5.3$ & $3.2 \pm 0.2$ & $30.7 \pm 19.0$ & $56.2 \pm 12.5$ & $3.8 \pm 1.4$ \\
\hline
\end{tabular}

PEM, pemetrexed; CDDP, cisplatin; GEM, gemcitabine.

chemotherapy while the other 3 patients were judged as having stable disease according to the Response Evaluation Criteria in Solid Tumors (RECIST). Written informed consent was obtained from all patients and the specimens of the patients were inspected according to the Declaration of Helsinki.

Growth inhibition assay. Growth inhibition was assessed by the MTS assay to examine the effect of PEM on MPM cell lines, as previously described (4). The $\mathrm{IC}_{50}$ value was calculated by SigmaPlot12 (Hulinks Inc., Tokyo, Japan). Experiments were performed independently 3 times.

RNA isolation and cDNA array and microarray data analysis. Total RNA was isolated from MPM cell lines with the use of TRIzol ${ }^{\circledR}$ reagent (Invitrogen, Carlsbad, CA), as previously described (5,6). High-density oligonucleotide array analysis was carried out using Affymetrix HG-U133A (22,282 probe sets) GeneChips, as previously described (7).

Quantitative real-time polymerase chain reaction analysis. Gene expression was examined by quantitative real-time PCR (qRT-PCR) analysis using a TaqMan Human Gene Expression Assay (Applied Biosystems, Foster City, CA). GAPDH served as an endogenous control. Gene expression data (mean \pm SD from triplicate samples) are shown as $\Delta \mathrm{CT}$.

Western blot analysis. Cells were lysed in buffer containing $50 \mathrm{mM}$ Tris- $\mathrm{HCl}$, pH 7.6, $150 \mathrm{mM} \mathrm{NaCl}, 0.1 \%$ sodium dodecyl sulfate, $1 \%$ Nonidet P-40, and $0.5 \%$ sodium deoxycholate. Western blot analysis was performed as previously described (6). TS and DHFR antibodies were kindly provided from Taiho Pharmaceutical Inc. (Tokyo, Japan). Antibodies detecting phosphorylated-AKT (p-AKT, Ser473), AKT and $\beta$-actin were purchased from Cell Signaling Technology (Beverly, MA).

Oligonucleotide transfection. Small interference RNAs (siRNAs) targeting SPP1 were purchased from Dharmacon Research Inc. (Lafayette, CO) and the homologous negative controls were obtained from Invitrogen. SPP1 siRNAs were transfected using Lipofectamine 2000 reagent $24 \mathrm{~h}$ after seeding, according to the manufacturer's instructions (Life Technologies, Carlsbad, CA). Transfections of siRNA complexes were added to cells at a final concentration of $50 \mathrm{nM}$.

Lentiviral-mediated SPP1-overexpressing cells. The coding sequence of human SPP1 or enhanced green fluorescent protein was inserted in expression plasmid vector pEZ-Lv151 (GeneCopoeia, Rockville, MD). Among several SPP1 isoforms, we chose the construct expressing SPP1-A (reference sequence M83248.1). These plasmids were cotransfected into 293Ta cells with the Lenti-Pac HIV Packaging Mix (GeneCopoeia). H2452 and MESO1 cells were transduced with serial dilutions of lentiviral supernatant in the presence of polybrene and selected by geniticine. After antibiotic selection for 3 weeks, stable SPP1-overexpressing cells were obtained.

Invasion assay. The ability of cells overexpressing SPP1 to invade through a Transwell was measured using cytoselect 96-well cell invasion assay according to the manufacturer's protocol (Cell Biolabs, Inc., San Diego, CA).

Statistical analysis. Data analysis for the correlation coefficients that revealed a correlation between the drug activity patterns and the gene expression patterns was principally done by a modified National Cancer Institute program (8). We used pathway analysis to provide a viewpoint of the biological function of genes within the proposed classifier (Stratagene, La Jolla, CA), as previously described (4).

Immunohistochemistry and scoring. SPP1/OPN protein expression was evaluated by immunohistochemistry (IHC) using an anti-human osteopontin mouse monoclonal antibody (Immuno-Biological Laboratories, Gunma, Japan). Immunohistochemical scoring was performed using the Histoscore (H-score) $(9,10)$. The expression of SPP1/OPN was estimated as the percentage of tumor cells with cytoplasmic immunoreactivity. Positive staining areas in the entire tissue section and the average intensity of the positive tumor cells were defined as follows. If more than $25 \%$ of the cells were stained, the specimen was considered to be positive for SPP1/OPN expression. The results of IHC were judged independently by two investigators (R.N. and F.Z) who were unaware of the clinical data, and consensus was reached for any discordant cases.

\section{Results}

Effect of PEM treatment on cell growth of MPM cells. We examined the antitumor activities of PEM against 6 MPM cell lines (Table I). The recommended serum level of PEM has been recognized as under $1 \mu \mathrm{M}$ in clinical trials of PEM. According to the sensitivity to PEM, these cell lines were classified as sensitive $\left(\mathrm{IC}_{50}\right.$ of $\left.\leq 1 \mu \mathrm{M}\right)$ or resistant $\left(\mathrm{IC}_{50}\right.$ of $\left.>1 \mu \mathrm{M}\right)$. The $\mathrm{H} 28$ 
A

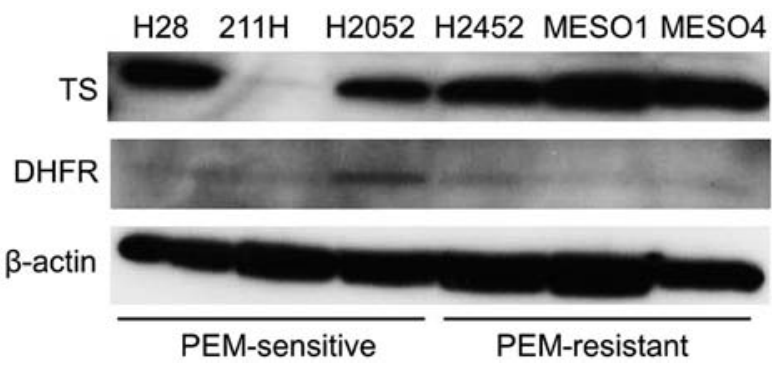

B

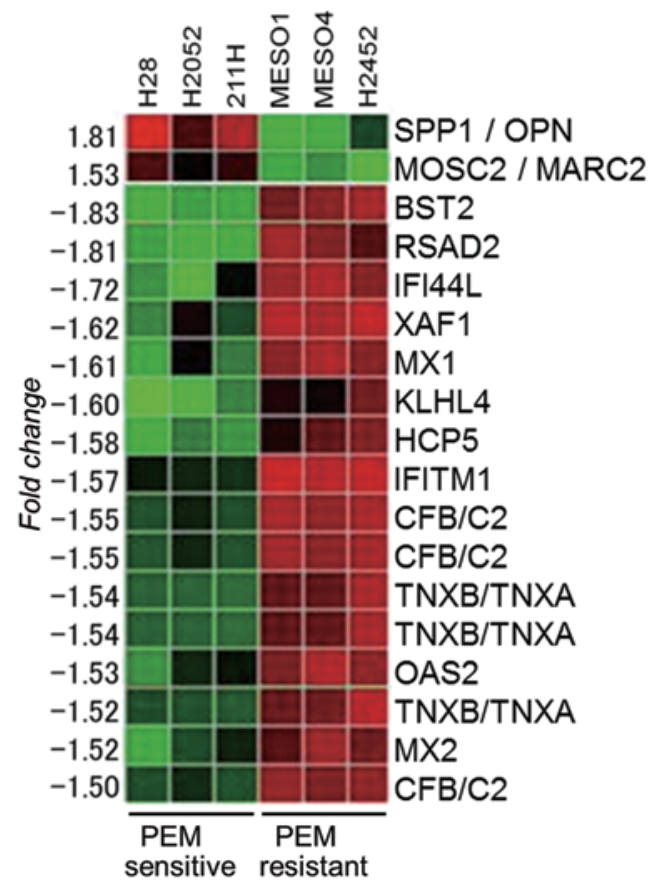

C

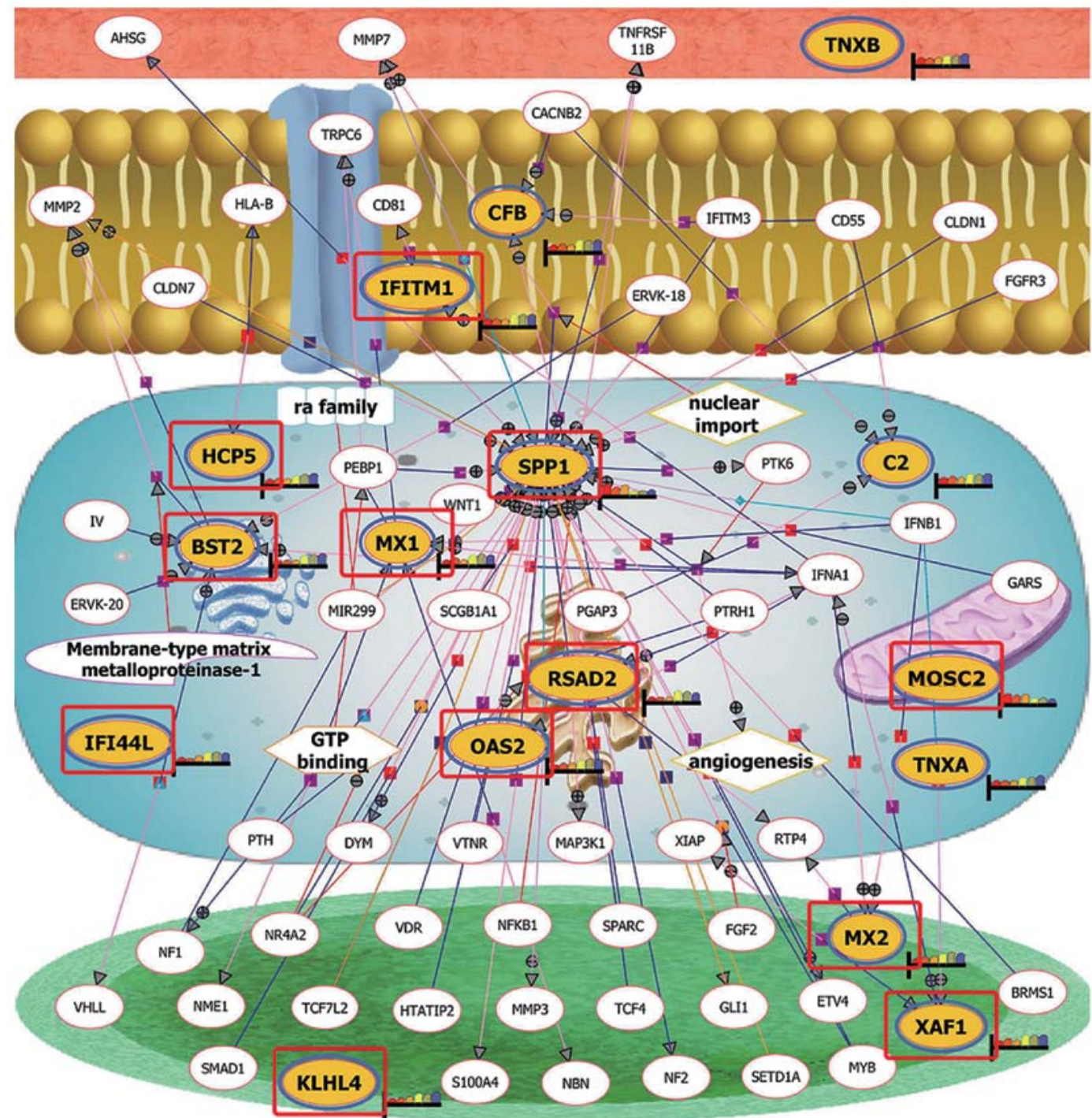

Figure 1. Genes that are differentially expressed between PEM-sensitive and resistant cells. (A) Protein expression of TS and DHFR was determined by western blot analyses. (B) Gene transcripts $(\mathrm{n}=18)$ and genes $(\mathrm{n}=14)$ were differentially expressed between three PEM-sensitive cell lines and three PEM-resistant cell lines. Fold change $>1.5$, p-value $<0.05$. (C) Twelve genes (red circle) associated with PEM responses were established by pathway analyses. 
D
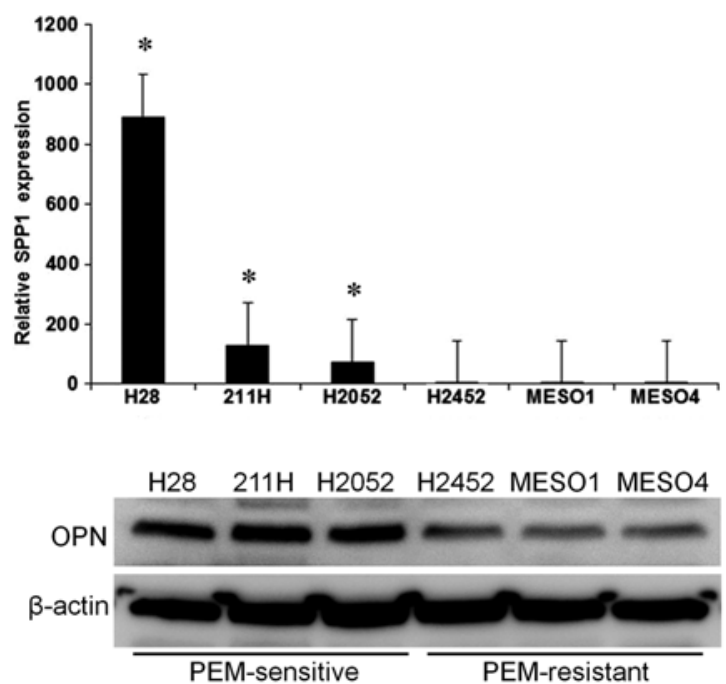

$\mathbf{E}$

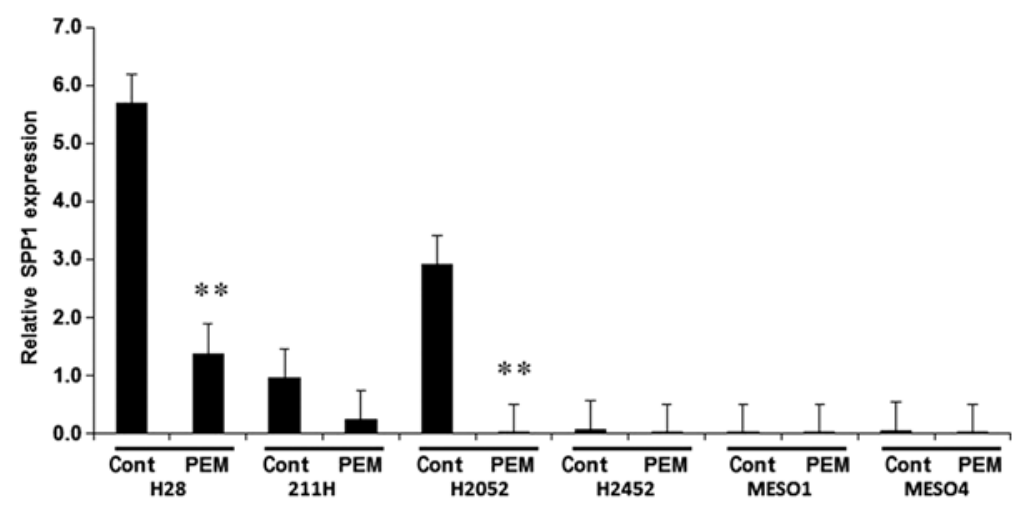

Figure 1. Continued. (D) SPP1/OPN gene expression level was significantly higher in the PEM-sensitive cell lines than in the resistant cell lines by quantitative RT-PCR (qRT-PCR) analysis ( ${ }^{*}$ p<0.05) (upper). Protein expression of osteopontin (OPN) was determined by western blot analyses (lower). (E) SPP1/OPN expression in two PEM-sensitive cell lines was significantly reduced in the presence of PEM $(1 \mu \mathrm{M})$ at $24 \mathrm{~h}$. Data are expressed as the mean \pm SD from 3 independent experiments. ${ }^{* *} \mathrm{p}<0.01$ when compared with the respective control cells (paired t-test).

and $211 \mathrm{H}$ cell lines had an $\mathrm{IC}_{50}$ of $<0.1 \mu \mathrm{M}$ (highly-sensitive). The $\mathrm{H} 2052$ cell line had an $\mathrm{IC}_{50}$ of 0.1 to $1 \mu \mathrm{M}$ (intermediatesensitive). The PEM-resistant group included H2452, MESO1 and MESO4 cells. In clinical trial data, PEM was shown to be effective in MPM patients with epithelial histological type. However, in this in vitro study, PEM had antitumor effects against MPM independent of the histological type. We confirmed that the sensitivity to PEM was independent of the sensitivities to CDDP and gemcitabine (GEM) (Table I).

PEM is a multi-target antifolate agent active on three enzymes involved in the synthesis of thymidine and purine nucleotides: thymidilate synthetase (TS), dihydrofolate reductase (DHFR), and glycinamide ribonucleotide formyl-transferase (GARFT) (11). We first examined the expression levels of TS and DHFR in the 6 MPM cell lines by western blot analysis. GARFT antibody was not commercially available. TS expression was absent in $211 \mathrm{H}$ cells (Fig. 1A). As for DHFR, only H2052 cells showed a weak band. Therefore, we assume that multiple mechanisms are involved in the sensitivity of MPM to PEM.

SPP1/OPN as a marker of the sensitivity of MPM to PEM. We performed gene expression profiling of the same set of 6 MPM cell lines by GeneChip analysis. A total of 18 transcripts and 14 genes were significantly correlated with the sensitivity to PEM (fold change $>1.5, \mathrm{p}<0.05$ ) (Fig. 1B). Twelve genes associated with chemosensitivity to PEM, were identified based on the biological functions of the altered/associated genes by pathway analysis (Fig. 1C) (4,7). Pathway analysis revealed that SPP1, which is also called osteopontin (OPN), was an important protein associated with the sensitivity of MPM cells to PEM.

We validated the upregulated SPP1/OPN gene expression and the downregulated expression of 9 genes in the 3 PEM-sensitive cell lines using commercially available qRT-PCR analysis (Fig. 1D). SPP1/OPN gene expression level in the PEM-sensitive cells was significantly higher than that in the PEM-resistant cells (Fig. 1D). Western blot analyses also showed that OPN protein expression was increased in the PEM-sensitive cell lines (Fig. 1D). We further evaluated the correlation between SPP1/OPN expression and PEM response in MPM cells. In 2 of the 3 PEM-sensitive cell lines with high SPP1/OPN, treatment with $1 \mu \mathrm{M}$ PEM significantly reduced the SPP1/OPN level (Fig. 1E). In the three resistant cell lines with low SPP1/OPN, PEM treatment did not affect SPP1/OPN expression (Fig. 1E). These results suggest that the SPP1/OPN gene is a candidate gene as a predictive marker of PEM sensitivity.

SPP1/OPN enhances invasion activity in MPM cells. To evaluate the biological function of SPP1/OPN in MPM cells, we developed a lentiviral vector for the expression of SPP1/OPN in the PEM-resistant cell lines that had low SPP1/OPN expression. We established stable SPP1-overexpressing cells (H2452-SPP1 and MESO1-SPP1). qRT-PCR analysis and western blot analysis showed remarkable overexpression of SPP1 in 3 SPP1 overexpressed cells (Fig. 2A). Introduction of SPP1 significantly enhanced the invasion activities of H2452 and MESO1 cells in the invasion assay (Fig. 2B). Next, we inhibited SPP1 expression using specific siRNAs in three PEM-sensitive cell lines with overexpressed SPP1/OPN (Fig. 2C). Inhibition of SPP1 using si-SPP1-A significantly suppressed the invasion activities of $211 \mathrm{H}$ cells in the invasion assay (Fig. 2C). These results suggest that SPP1/OPN enhanced the cell invasiveness of MPM cells.

Effect of SPPI/OPN expression on PEM sensitivity via $A K T$ signaling pathway. A recent report demonstrated that SPP1/OPN provided MPM cells with multidrug resistance via upregulation of the AKT signaling pathway (12). We found that SPP1 overexpression by lentivirus induced the elevation of AKT phosphorylation ( $\mathrm{p}-\mathrm{AKT}$ ) in MESO1-SPP1 cells 
$\mathbf{A}$

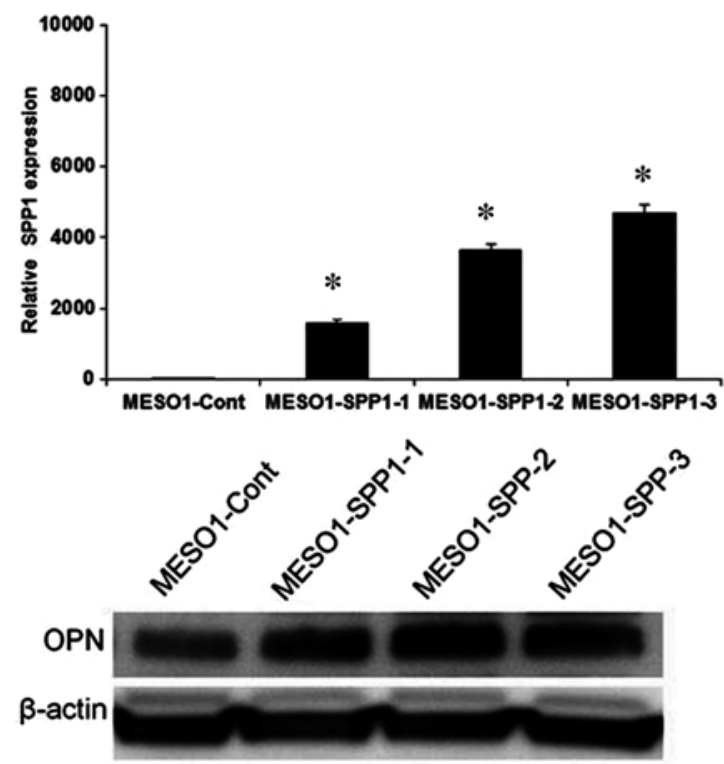

B
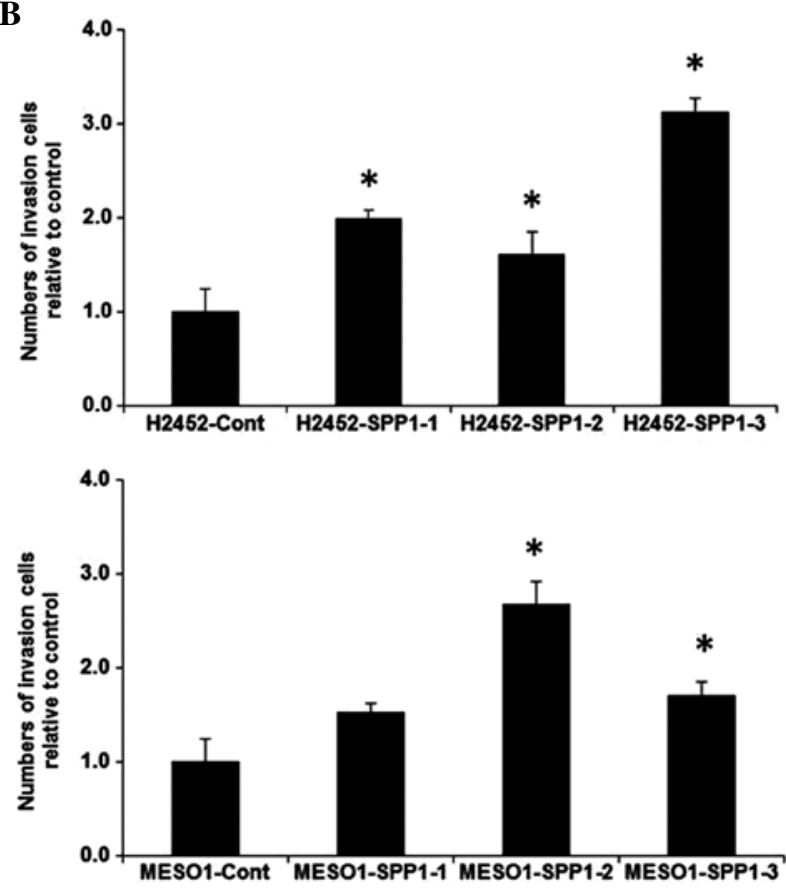

C
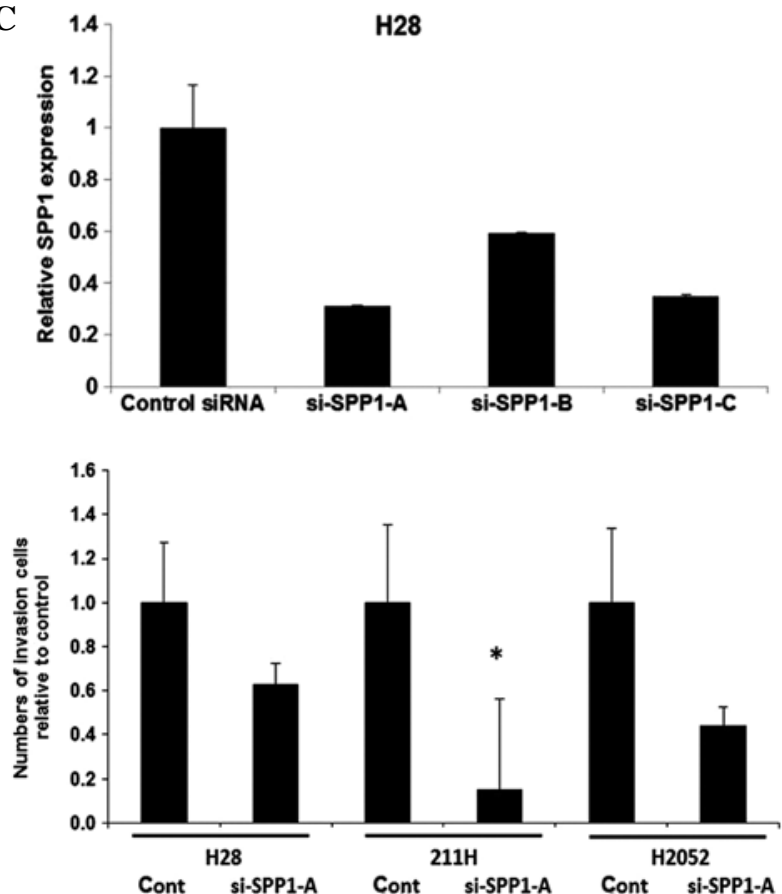

Figure 2. Effect of SPP1/OPN expression on invasion activity. (A) SPP1 expression levels in MESO1 cells with lentiviral-mediated production of SPP1 (MESO1-SPP1). The SPP1 expression levels in three MESO1-SPP1 cell lines were significantly higher than those in the control MESO1 cells by qRT-PCR analysis (upper) and western blot analyses (lower). (B) In vitro invasion assay of H2452-SPP1 cells and MESO1-SPP1 cells. Invasion activities of H2452-SPP1 cells and MESO1-SPP1 cells were significantly increased compared with those in H2452 and MESO1 cells, as measured by invasion assay. (C) SPP1 inhibition by si-SPP1 in $\mathrm{H} 28$ cells. Expression of SPP1 was significantly reduced by multiple si-SPP1 up to $72 \mathrm{~h}$ in H28 cells (upper). In vitro invasion assay of three PEMsensitive cell lines after the transfection of siSPP1-A for $24 \mathrm{~h}$ (lower). Inhibition of SPP1 significantly suppressed the invasion activities of $211 \mathrm{H}$ cells (upper). Data are expressed as the mean \pm SD from 3 independent experiments. " $p<0.05$ when compared with the respective control cells (paired $t$-test).

(Fig. 3A). On the other hand, inhibition of SPP1 in H28 cells with high SPP1 could reduce p-AKT and AKT expression (Fig. 3B). The levels of AKT and p-AKT did not change in MESO1 cells with a low level of SPP1 by SPP1 siRNA (Fig. 3B). We further evaluated the correlation between AKT activation and PEM response in MPM cells. After PEM treatment of PEM-sensitive H28 cells, the p-AKT level significantly decreased (Fig. 3C). In contrast, PEM treatment did not change the p-AKT and AKT levels in the PEM-resistant
MESO1 cells with low SPP1 (Fig. 3C). To further evaluate the significance of SPP1/OPN and AKT activation in the PEM-induced cell growth inhibitory effect, activation of phosphoinositide 3-kinase (PI3K)/AKT signaling pathway was examined in these MPM cells. We analyzed the effect of a well-known potent and selective PI3K inhibitor, wortmannin, on cell growth activity in MPM cells. The p-AKT level in $\mathrm{H} 28$ and MESO1 cells significantly decreased after incubation with wortmannin (1-10 $\mu \mathrm{M})$ (Fig. 3D). We cotreated H28 
A

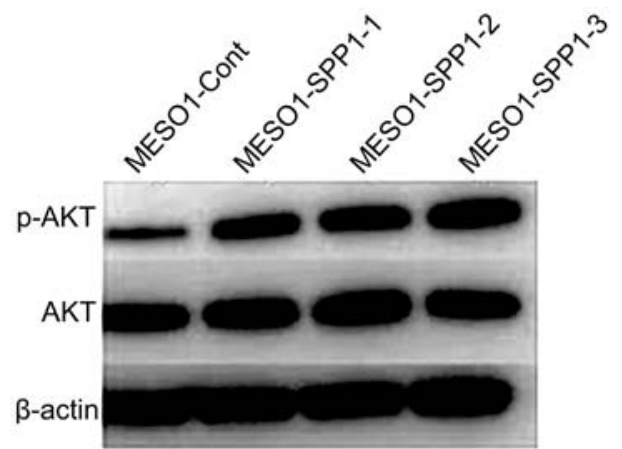

C

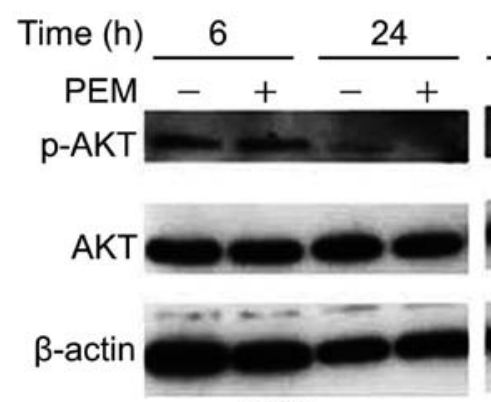

$\mathrm{H} 28$
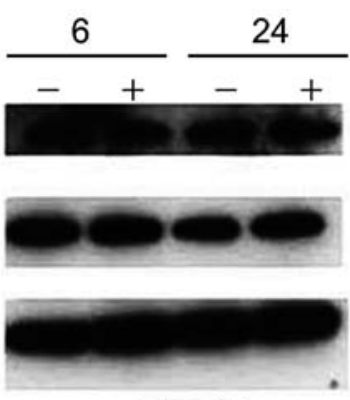

MESO1
$\mathbf{E}$

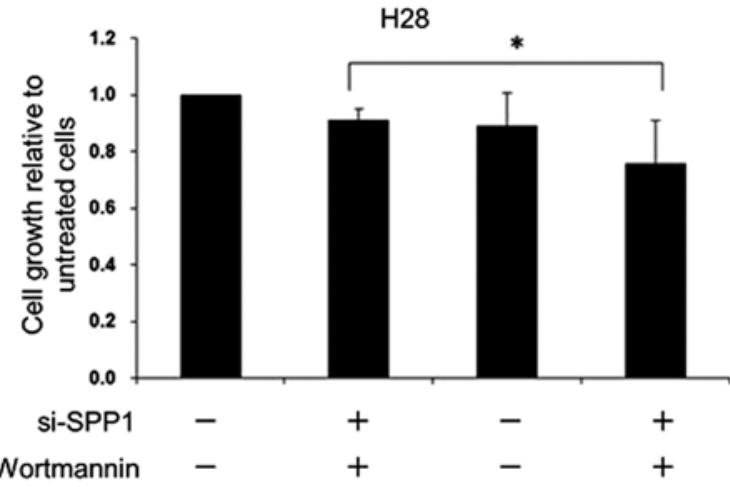

$\mathrm{H} 28$

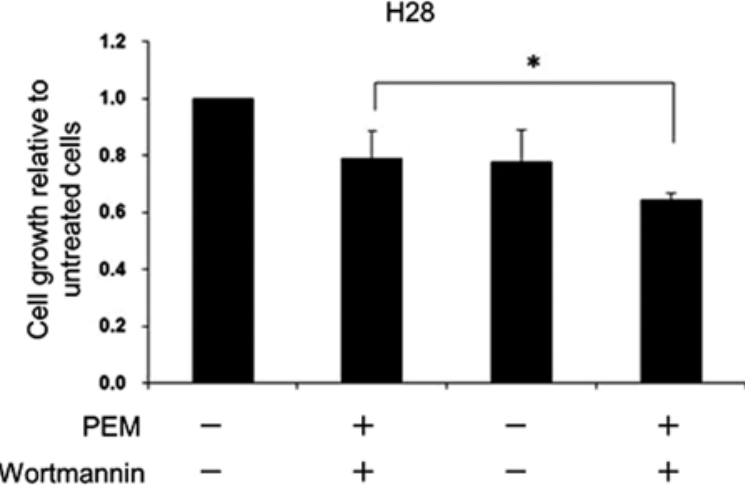

MESO1

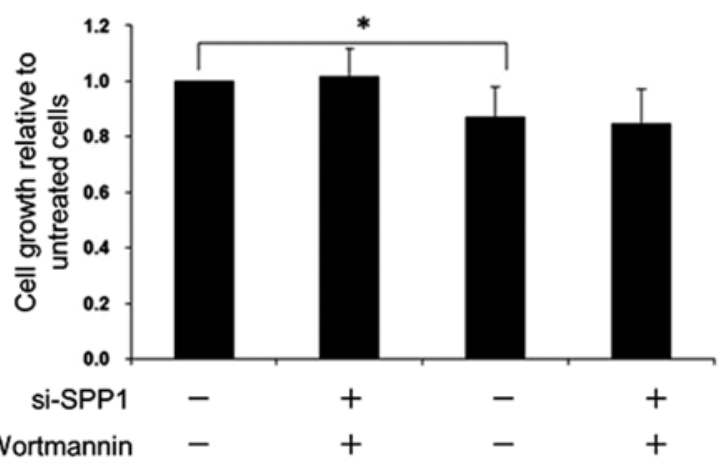

B

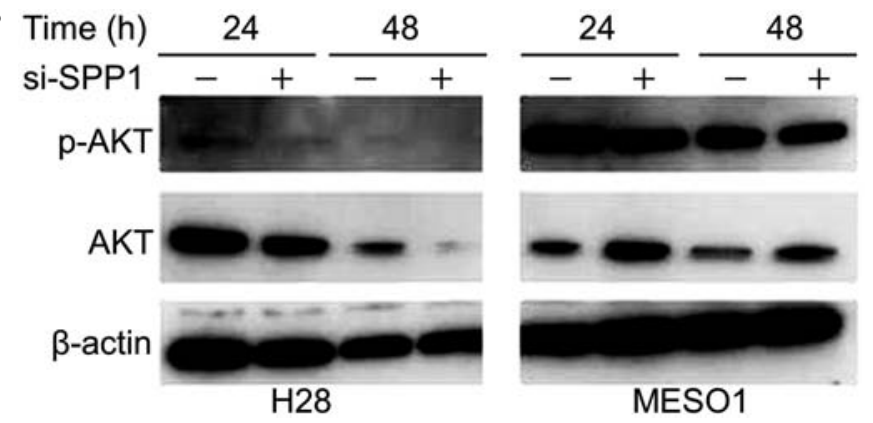

D
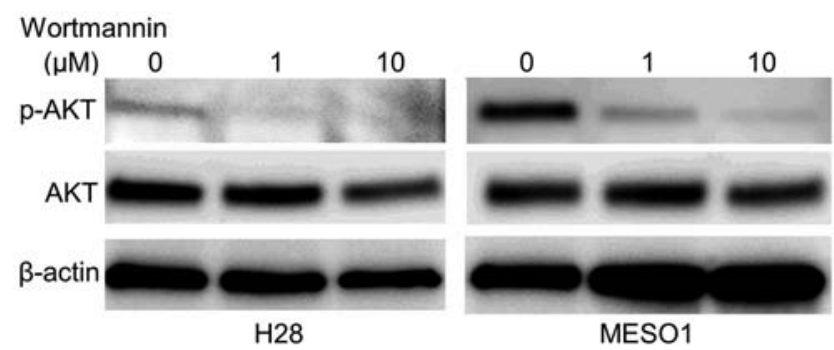

$\mathbf{F}$

PEM-sensitive cells

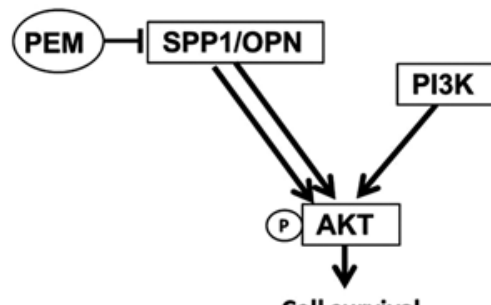

Cell survival

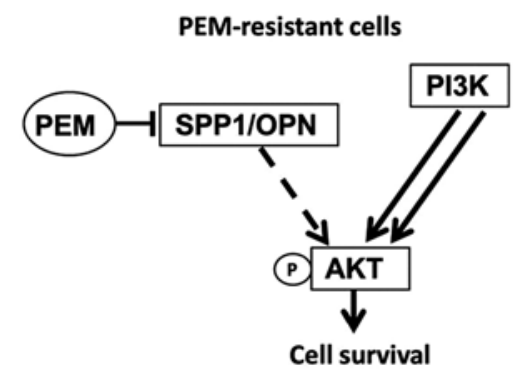

Figure 3. Effect of SPP1/OPN on PEM sensitivity via the AKT pathway. (A) AKT and p-AKT levels in MESO1-SPP1 cells with lentiviral-mediated production of SPP1. The levels of AKT and p-AKT in three MESO-SPP1 cell lines were significantly higher than those in the control MESO1 cells by western blot analysis. (B) SPP1 inhibition by siSPP1 reduced AKT and p-AKT levels in H28 cells from 24 to $48 \mathrm{~h}$. SPP1 suppression could reduce p-AKT and AKT levels in $\mathrm{H} 28$ cells with high SPP1. (C) AKT and p-AKT levels in H28 and MESO1 cells from 6 to $24 \mathrm{~h}$ after treatment with PEM $(1 \mu \mathrm{M})$. The p-AKT level significantly decreased after PEM treatment of PEM-sensitive H28 cells. (D) Wortmannin decreased p-AKT protein levels in H28 and MESO1 cells. MPM cells were exposed to wortmannin $(1-10 \mu \mathrm{M})$ for $24 \mathrm{~h}$. After the treatment, the cells were subjected to western blot analysis. (E) Effect of combination therapy with wortmannin and SPP1 silencing or PEM on cell growth in MPM cells. Wortmannin $(10 \mu \mathrm{M})$ treatments with PEM $(1 \mu \mathrm{M})$ or siSPP1 $(50 \mathrm{nM})$ for $48 \mathrm{~h}$ was examined in $\mathrm{H} 28$ cells (upper panel). Wortmannin $(10 \mu \mathrm{M})$ with siSPP1 $(50 \mathrm{nM})$ for $48 \mathrm{~h}$ was examined in MESO1 cells (lower panel). Each result is expressed as cell viability in treated cells compared with the siSPP1-transfected cells or the untreated cells $(100 \%)$. Data are expressed as the mean \pm SD from 3 independent experiments. ${ }^{p}<0.05$ when compared with the siSPP1-transfected cells or the untreated cells (paired t-test). (F) Putative mechanism of the sensitivity of MPM cells to PEM. AKT activation induced by SPP1/OPN is critical for the survival of PEM-sensitive MPM cells (lower). Constitutive PI3K/AKT activation without SPP1/OPN overexpression contributes to cell survival in PEM-resistant cells (upper). 
G

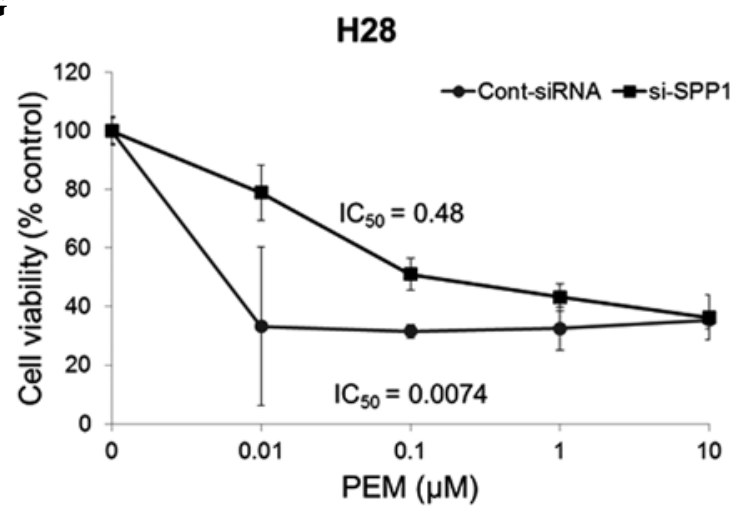

211H

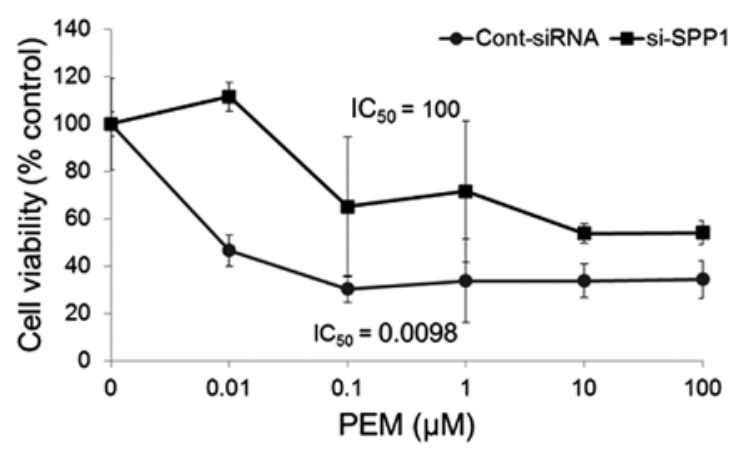

H2052

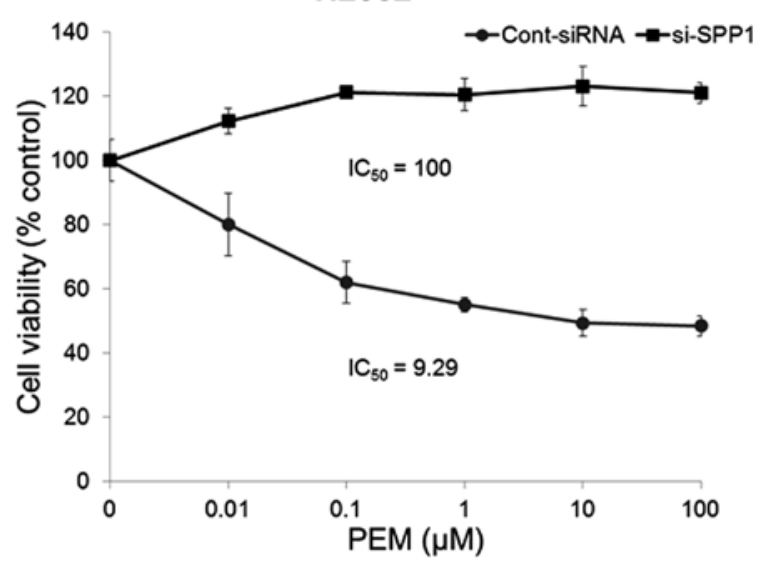

Figure 3. Continued. (G) Effect of SPP1 expression on the sensitivity of MPM cells to PEM. siSPP1 was transfected in three PEM-sensitive MPM cell lines for 24 hours. Then, the effect of PEM treatment for 72 hours was examined in the three cell lines. Each result is expressed as cell viability compared with that in the untreated sample $(100 \%)$.

cells with wortmannin and siSPP1 or PEM, and then evaluated cell growth activity. Combination treatment of wortmannin with PEM or siSPP1 significantly reduced cell numbers in $\mathrm{H} 28$ cells with SPP1/OPN overexpression (Fig. 3E). In contrast, wortmannin could reduce cell growth in MESO1 cells; however, a synergic effect of wortmannin and siSPP1 in MESO1 with low SPP1/OPN expression was not observed (Fig. 3E). These results suggest that SPP1/OPN through AKT activation contributes to PEM sensitivity in PEM-sensitive cell lines with overexpressed SPP1/OPN (Fig. 3F). In the resistant cells, constitutive $\mathrm{PI} 3 \mathrm{~K} / \mathrm{AKT}$ activation might be dominantly involved in the resistance to PEM (Fig. 3F).
A

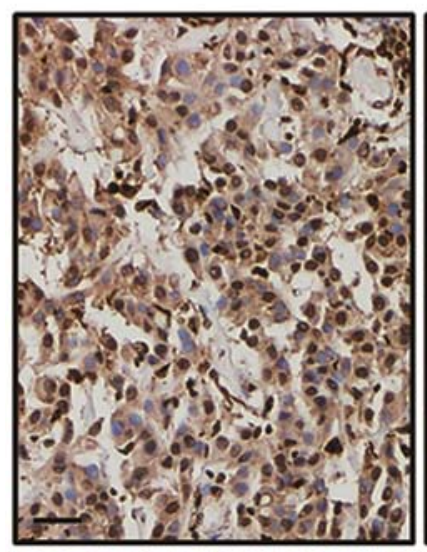

B
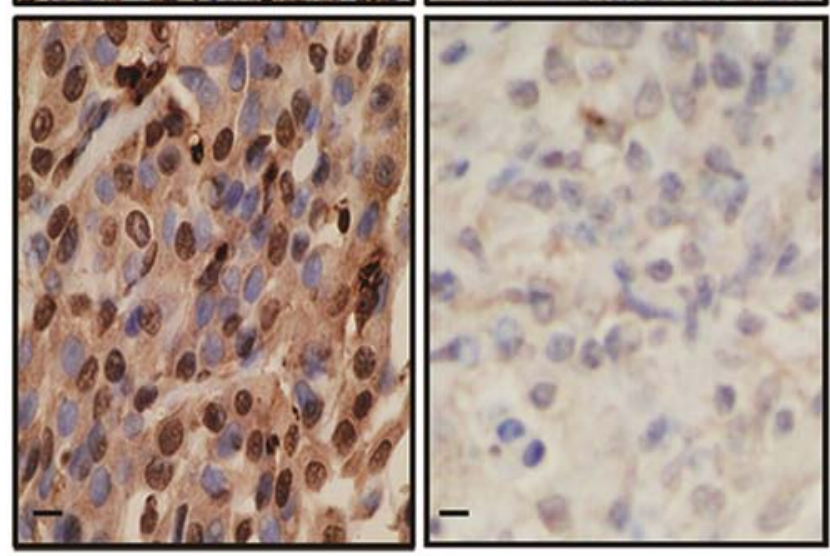

Figure 4. Immunohistochemical staining of SPP1/OPN in MPM tissues. (A) High level of SPP1/OPN in MPM (patient 2). (B) Low level of SPP1/OPN in MPM (patient 4). Upper row bars, $100 \mu \mathrm{m}$; and lower row bars, $10 \mu \mathrm{m}$.

We further investigated the effect of SPP1/OPN on the sensitivity of MPM to PEM.PEM treatment after suppression of SPP1/ OPN by siSPP1 transfection diminished the growth-inhibitory effect of PEM in three PEM-sensitive MPM cell lines (Fig. 3G). The $\mathrm{IC}_{50}$ values of PEM with si-SPP1 treatment of $\mathrm{H} 28,211 \mathrm{H}$ and $\mathrm{H} 2052$ cells were $0.48,>100 \mu \mathrm{M}$ and $>100 \mu \mathrm{M}$, respectively, whereas the $\mathrm{IC}_{50}$ values of PEM in these 3 cells without si-SPP1 treatment were 0.007,0.01 and 9.3 $\mu \mathrm{M}$, respectively (Fig. 3G). In addition, the growth-inhibitory effect of PEM on H2452-SPP1 and MESO1-SPP1 cells was assessed by MTS assay. The drug sensitivities were unaffected in stable SPP1-overexpressing H2452 and MESO-1 cells (data not shown). These results indicate that SPP1/OPN expression contributes to the sensitivity of MPM cells to PEM. Therefore, SPP1/OPN could be used as a marker of the sensitivity of MPM cells to PEM.

Expression level of SPP1/OPN in MPM specimens. In order to evaluate the clinical application of SPP1/OPN as a predictive marker, we examined the expression level of SPP1/OPN in tumor sections by IHC and evaluated the relationship between the expression of SPP1/OPN and the tumor response to PEM treatment with platinum agents. Two of three MPM patients judged as having a PR to chemotherapy were positive for SPP1/OPN (Fig. 4A, Table II). In contrast, no positive staining was observed in MPM patients judged as having stable disease (SD) (Fig. 4B, Table II). 
Table II. Characteristics of MPM patients treated with PEM and results of OPN staining.

\begin{tabular}{lclllcrc}
\hline Sample & $\begin{array}{c}\text { Age } \\
\text { (years) }\end{array}$ & Gender & $\begin{array}{c}\text { Pathological } \\
\text { type }\end{array}$ & Regimen & $\begin{array}{c}\text { No. of } \\
\text { courses }\end{array}$ & RECIST & $\begin{array}{c}\text { OPN } \\
\text { staining }\end{array}$ \\
\hline 1 & 71 & Female & Epithelial type & CDDP+PEM & 4 & PR & Positive \\
2 & 62 & Male & Epithelial type & CDDP+PEM & 6 & PR & Positive \\
3 & 77 & Male & Sarcoma type & CBDCA+PEM & 4 & PR & Negative \\
4 & 56 & Male & Epithelial type & CDDP+PEM & 3 & SD & Negative \\
5 & 75 & Male & Sarcoma type & CBDCA+PEM & 2 & SD & Negative \\
6 & 67 & Male & Epithelial type & CBDCA+PEM & 4 & SD & Negative \\
\hline
\end{tabular}

PR, partial response; $\mathrm{SD}$, stable disease.

\section{Discussion}

PEM is a multi-target antifolate agent active on three enzymes involved in the synthesis of TS, DHFR and GARFT (11). Among them, TS is well known as a predictive marker for the sensitivity to antifolate drugs including PEM. High TS expression was significantly associated with resistance to PEM in NSCLC cells (13). In addition, a previous phase II study suggested the potential association between low TS expression and response to PEM therapy in MPM (14). However, no relationship between low TS expression and sensitivity to PEM was found among the MPM cell lines in the present study. In fact, 2 cell lines, H28 and H2052, had high TS expression among the 3 PEM-sensitive MPM cell lines investigated in this study. A recent report demonstrated that PEM sensitivity was frequently associated with qualitative and/or quantitative alterations in influx and/or efflux transporters of antifolates as well as in folate-dependent enzyme (15). In MPM, multiple mechanisms including TS expression are implicated in the response to PEM.

SPP1/OPN is a glycoprotein and functions as both an extracellular matrix (ECM) component and cytokine through binding to its receptors, integrin and CD44 (16). SPP1/OPN has been associated with human cancer progression, metastasis and apoptosis (17). Overexpression of SPP1/OPN has been observed in a variety of human malignant tumors, including lung, breast, prostate, colon, ovarian and gastric cancers (18-23). Previously it was reported that elevated levels of SPP1/OPN in serum and pleural effusion have been observed in MPM patients and SPP1/OPN was recognized as a differential diagnostic biomarker $(24,25)$. High SPP1/OPN level was an independent negative predictor of survival in MPM (26). These results suggested that SPP1/OPN is actually involved in the pathogenesis of MPM and can be used as a diagnostic and prognostic marker of MPM.

In this study, we demonstrated that SPP1/OPN could be used as a predictive marker of the sensitivity of MPM to PEM. The expression level of SPP1/OPN in PEM-sensitive cells was significantly higher than that in PEM-resistant cells. We confirmed that high expression levels of SPP1/OPN were observed in 2 of 3 MPM patients who had a PR to PEM treatment with platinum agents. Our study is the first to report that SPP1/OPN can be useful as a predictive marker for the effectiveness of PEM therapy in MPM. A recent report described that MPM cells overexpressing SPP1/OPN were more resistant to various cytotoxic drugs than the parent cells through the mechanism of enhanced hyaluronate and CD44 (12). CD44-mediated resistance to apoptosis induced by the cytotoxic drugs was dependent on activation of the AKT pathway. In this study, overexpressed SPP1/OPN enhanced the level of p-AKT in MPM cells. In PEM-sensitive MPM cells, PEM treatment significantly reduced SPP1 expression, resulting in a reduced level of $\mathrm{p}-\mathrm{AKT}$. These findings suggest that AKT activation induced by SPP1/OPN is critical for the survival of PEM-sensitive MPM cells. On the other hand, in PEM-resistant MPM cells, constitutive AKT activation regulated by another mechanism including PI3K signal may contribute to resistance to PEM. Cell growth of these resistant cells is independent of the SPP1/OPN-induced AKT signaling pathway. These results suggest that PEM has antitumor effects in MPM cells dependent on SPP1/OPN activation. Tajima et al (12) reported that MPM cells that overexpressed OPN showed resistance to cytotoxic agents including GEM, vinorelbin and etoposide. However, these OPN-overexpressing cells did not show resistance to CDDP. In our analyses, the 3 PEM-sensitive cell lines did not show strong resistance to CDDP compared to the resistant cell lines. Therefore, the combination therapy of PEM with CDDP may have clinical benefit in MPM patients with overexpressed SPP1/OPN. One limitation of our study was that the number of patient samples was very small; however, these results confirmed that the IHC expression level of SPP1/OPN could be predictive of the response to PEM-based chemotherapy in MPM patients.

In addition to SPP1/OPN, we identified 17 gene transcripts that were significantly correlated with sensitivity to PEM. Three of the 17 gene transcripts were identified as tenascin $\mathrm{XA} /$ tenascin XB (TNXA/TNXB) genes located in the ECM and their expression was significantly increased in PEM-resistant cells. TNXB was previously identified as a gene that is more highly expressed in malignant mesothelioma and a candidate diagnostic marker of malignant mesothelioma (27). Although the association between low level of SPP1/OPN and high expression of TNXA/TNXB in PEM-resistant cells is unclear, alteration of genes encoding ECM proteins, SPP1/OPN and TNXA/TNXB, may be critical to the carcinogenesis of MPM and sensitivity to PEM.

In conclusion, PEM has antitumor effects in MPM cells dependent on SPP1/OPN overexpression resulting in AKT activation. Our results suggest that SPP1/OPN may be used as a single predictive biomarker of the effectiveness of PEM treatment in MPM. 


\section{Acknowledgements}

This study was supported in part by a Grant-in-Aid from the Ministry of Education, Culture, Sports, Science and Technology of Japan, the National Cancer Center Research and Development Fund (23-A-11) and MEXT-Supported Program for the Strategic Research Foundation at Private Universities (to M.S. and A.G.).

\section{References}

1. Hansen J, de Klerk NH, Musk AW and Hobbs MS: Environmental exposure to crocidolite and mesothelioma: exposure-response relationships. Am J Respir Crit Care Med 157: 69-75, 1998.

2. Robinson BWS and Lake RA: Advances in malignant mesothelioma. N Engl J Med 353: 1591-1603, 2005.

3. Vogelzang NJ, Rusthoven JJ, Symanowski J, et al: Phase III study of PEM in combination with cisplatin versus cisplatin alone in patients with malignant pleural mesothelioma. J Clin Oncol 21: 2636-2644, 2003.

4. Shimokawa T, Seike M, Gemma A, et al: Enzastaurin has anti-tumour effects in lung cancers with overexpressed JAK pathway molecules. Br J Cancer 106: 867-875, 2012.

5. Seike M, Yanaihara N, Bowman ED, et al: Use of a cytokine gene expression signature in lung adenocarcinoma and the surrounding tissue as a prognostic classifier. J Natl Cancer Inst 15: 1257-1269, 2007.

6. Seike M, Goto A, Harris CC, et al: MiR-21 is an EGFRregulated anti-apoptotic factor in lung cancer in never-smokers Proc Natl Acad Sci USA 21: 12085-12090, 2009.

7. Gemma A, Li C, Kudoh S, et al: Anticancer drug clustering in lung cancer based on gene expression profiles and sensitivity database. BMC Cancer 6: 174, 2006.

8. Miyanaga A, Gemma A, Kudoh S, et al: Antitumor activity of histone deacetylase inhibitors in non-small cell lung cancer cells: development of a molecular predictive model. Mol Cancer Ther 7: 1923-1930, 2008.

9. Kikuchi S, Honda K, Yamada T, et al: Expression and gene amplification ofactinin-4 in invasive ductal carcinoma of the pancreas. Clin Cancer Res 14: 5348-5356, 2008.

10. McCarty KS Jr, Miller LS, Cox EB, Konrath J and McCarty KS Sr: Estrogen receptor analyses. Correlation of biochemical and immunohistochemical methods using monoclonal antireceptor antibodies. Arch Pathol Lab Med 109: 716-721, 1985

11. Shih C, Chen VJ, Gossett LS, et al: LY231514, a pyrrolo [2,3-d] pyrimidine-based antifolate that inhibits multiple folaterequiring enzymes. Cancer Res 57: 1116-1123, 1997.

12. Tajima K, Ohashi R, Sekido Y, et al: Osteopontin-mediated enhanced hyaluronan binding induces multidrug resistance in mesothelioma cells. Oncogene 29: 1941-1951, 2010.
13. Ozasa H, Oguri T, Uemura T, Miyazaki M, Maeno K, Sato S and Ueda R: Significance of thymidylate synthase for resistance to PEM in lung cancer. Cancer Sci 101: 161-166, 2010.

14. Righi L, Papotti MG, Ceppi P, et al: Thymidylate synthase but not excision repair cross-complementation group 1 tumor expression predicts outcome in patients with malignant pleural mesothelioma treated with PEM-based chemotherapy. J Clin Oncol 28: 1534-1539, 2010.

15. Assaraf YG: Molecular basis of antifolate resistance. Cancer Metastasis Rev 26: 153-181, 2011.

16. Baliga SS, Merrill GF, Shinohara ML and Denhardt DT: Osteopontin expression during early cerebral ischemiareperfusion in rats: enhanced expression in the right cortex is suppressed by acetaminophen. PLoS One 6: e14568, 2011.

17. Rangaswami H, Bulbule A and Kundu GC: Osteopontin: role in cell signaling and cancer progression. Trends Cell Biol 16: 79-87, 2006.

18. Donati V, Boldrini L, Fontanini G, et al: Osteopontin expression and prognostic significance in non-small cell lung cancer. Clin Cancer Res 18: 6459-6465, 2005.

19. Tuck AB and Chambers AF. The role of osteopontin in breast cancer: clinical and experimental studies. J Mammary Gland Biol Neoplasia 6: 419-429, 2001.

20. Thalmann GN, Sikes RA, Devoll RE, et al: Osteopontin: possible role in prostate cancer progression. Clin Cancer Res 5: 2271-2277, 1999.

21. Agrawal D, Chen T, Irby R, et al: Osteopontin identified as lead marker of colon cancer progression, using pooled sample expression profiling. J Natl Cancer Inst 94: 513-521, 2002.

22. Kim J-H, Skates SJ, Uede T, et al: Osteopontin as a potential diagnostic biomarker for ovarian cancer. JAMA 287: 1671-1679, 2002.

23. Ue T, Yokozaki H, Kitadai Y, Yamamoto S, Yasui W, Ishikawa T and Tahara E: Co-expression of osteopontin and CD44v9 in gastric cancer. Int J Cancer 79: 127-132, 1998.

24. Pass HI, Lott D, Lonardo F, et al: Asbestos exposure, pleural mesothelioma, and serum osteopontin levels. N Engl J Med 353: 1564-1573, 2005.

25. Grigoriu BD, Scherpereel A, Devos P, et al: Utility of osteopontin and serum mesothelin in malignant pleural mesothelioma diagnosis and prognosis assessment. Clin Cancer Res 13: 2928-2935, 2007.

26. Hollevoet K, Nackaerts K, Meerbeeck JP, et al: Soluble mesothelin, megakaryocyte potentiating factor, and osteopontin as markers of patient response and outcome in mesothelioma. J Thorac Oncol 11: 1930-1937, 2011.

27. Yuan Y, Nymoen DA, Stavnes HT, et al: Tenascin-X is a novel diagnostic marker of malignant mesothelioma. Am J Surg Pathol 33: 1673-1682, 2009. 